1 TITLE: LSTrAP-Kingdom: an automated pipeline to generate annotated gene

2 expression atlases for kingdoms of life

3 AUTHORS: William Goh ${ }^{1}$, Marek Mutwil ${ }^{1 *}$

$5{ }^{1}$ School of Biological Sciences, Nanyang Technological University, 60 Nanyang Drive,

6 Singapore, 637551, Singapore

$8 \quad{ }^{*}$ Corresponding author:

9 Marek Mutwil

10 School of Biological Sciences,

11 Nanyang Technological University,60 Nanyang Drive,

12 637551, Singapore,

13 Singapore

14 Email: mutwil@ntu.edu.sg

\title{
16 Abstract
}

17 Summary: There are now more than two million RNA sequencing experiments for 18 plants, animals, bacteria and fungi publicly available, allowing us to study gene 19 expression within and across species and kingdoms. However, the tools allowing the 20 download, quality control and annotation of this data for more than one species at a 21 time are currently missing. To remedy this, we present the Large-Scale Transcriptomic 22 Analysis Pipeline in Kingdom of Life (LSTrAP-Kingdom) pipeline, which we used to 23 process 134,521 RNA-seq samples, achieving $\sim 12,000$ processed samples per day. 
24 Our pipeline generated quality-controlled, annotated gene expression matrices that rival

25 the manually curated gene expression data in identifying functionally-related genes.

Availability and implementation: LSTrAP-Kingdom is available from:

28 https://github.com/wirriamm/plants-pipeline and is fully implemented in Python and 29 Bash.

\section{Introduction}

32 When first developed, the main application of RNA sequencing (RNA-seq) was for 33 performing differential gene expression between samples (Stark et al., 2019). The 34 decreasing cost of sequencing and computational power has driven the explosive 35 growth of RNA-seq data (https://www.genome.gov/about-genomics/fact-sheets/DNA36 Sequencing-Costs-Data), evident in the increase in the amount of open access data on 37 Sequence Read Archive (SRA) from 100 Terabases in 2011 (Kodama et al., 2012), to 38 more than 10,000 Terabases in 2020

39 (https://www.ncbi.nlm.nih.gov/sra/docs/sragrowth/). The RNA-seq data is especially 40 abundant for animals $(1,774,720$ samples), followed by plants $(173,663)$, fungi $(53,712)$, 41 and bacteria $(50,615)$, at the time of writing of this manuscript. The RNA-seq data 42 allows coexpression analyses useful in gene function prediction and to supplement 43 integrated multi-omics analyses (Usadel et al., 2009; Rhee and Mutwil, 2014). 44 Furthermore, when combined with genomic information, comparative transcriptomic 45 analyses across species allow the study of the function and evolution of genes from the 46 perspective of gene expression (Ferrari et al., 2019; Wen Tan and Mutwil, 2019; Ferrari 47 et al., 2020; $\mathrm{Ng}$ et al., 2019; Ferrari and Mutwil, 2019; Lim et al., 2020).

51 currently not available. To remedy this paucity, we constructed an open-source pipeline, 
52 Large-Scale Transcriptomic Analysis Pipeline in Kingdom of Life (LSTrAP-Kingdom),

53 that allows rapid generation of kingdom-wide expression atlases. We used our

54 automated pipeline to download 134,521 RNA-seq experiments for 116 species,

55 achieving processing speed of $\sim 12,000$ samples per day, and show that the

56 experiments can be annotated with a simple natural language processing pipeline that

57 leverages organ ontology information. Finally, we show that the coexpression networks

58 obtained by our pipeline perform as well as networks constructed from manually 59 assembled matrices.
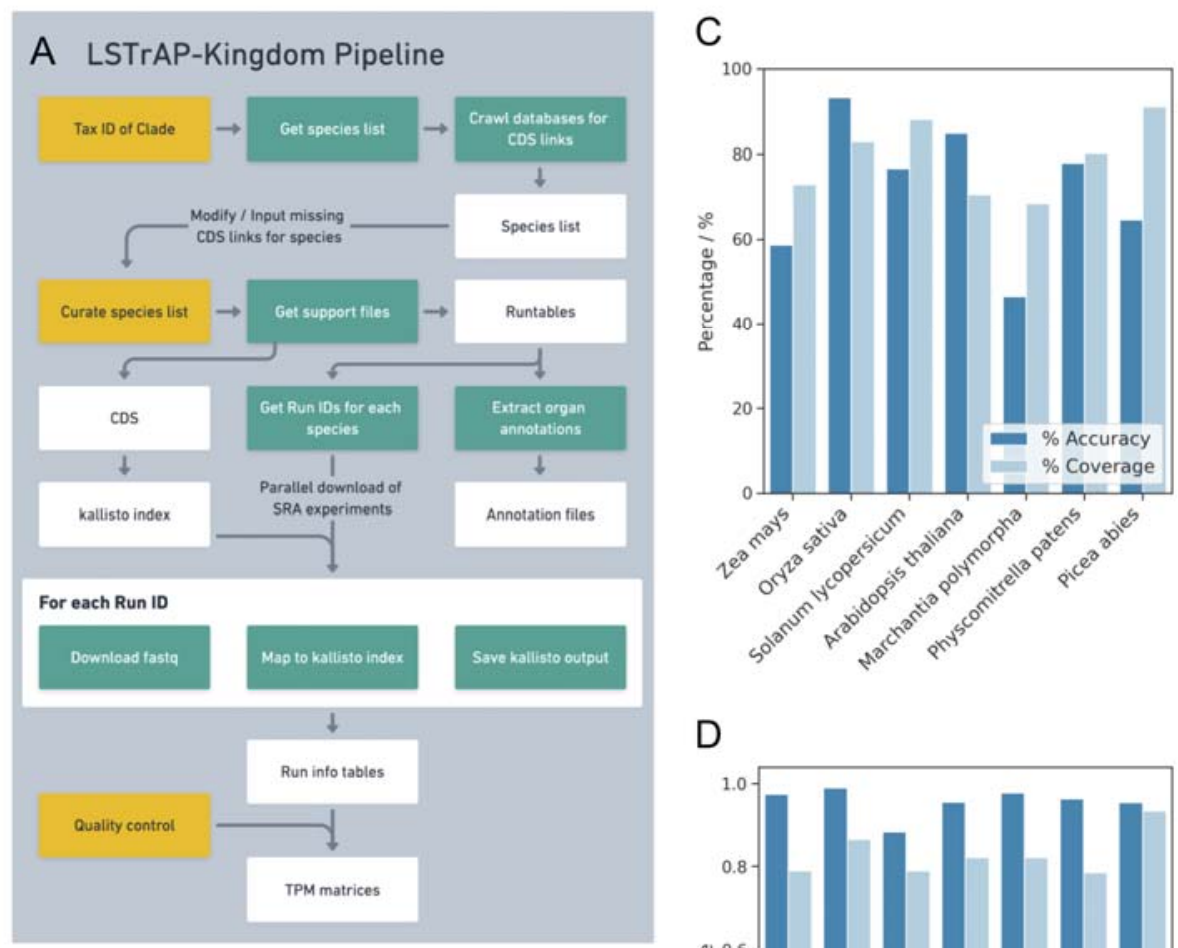

D
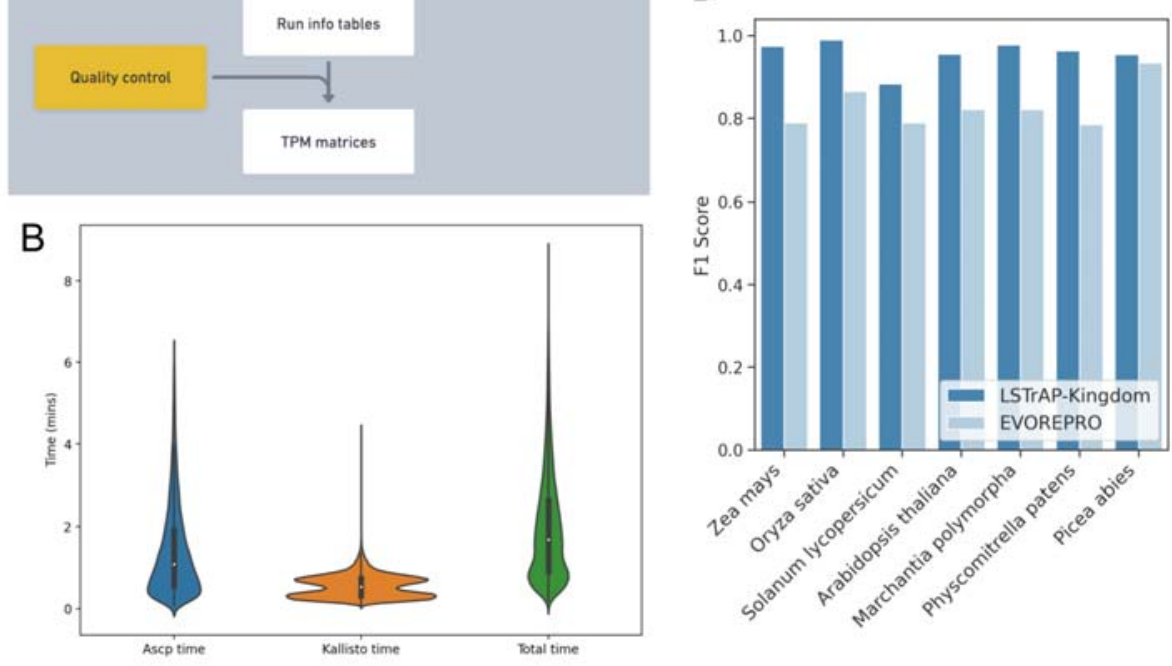

61 Figure 1. The LSTrAP-Kingdom pipeline. A) An outline of the pipeline. Yellow: user 62 input; green: processes; white: output files. B) Distribution of time taken (in minutes) for 
63 ascp download, Kallisto processing, and total time for 35,020 samples from Arabidopsis

64 thaliana. C) Percentage accuracy and coverage of LSTrAP-Kingdom annotation 65 benchmarked against the manually annotated samples from the EVOREPRO study. D)

66 F1 scores of coexpression networks generated by LSTrAP-Kingdom in predicting genes

67 for biosynthesis of ribosomal proteins, benchmarked against the EVOREPRO dataset.

682 Automated retrieval of coding sequences, available experiments, and gene expression value estimation

70 To demonstrate our pipeline, we used Viridiplantae (taxid 33090), encompassing all

71 green plants (Figure 1A). LSTrAP-Kingdom retrieves taxonomic IDs (taxid) for all

72 species found under the clade of interest from NCBI Taxonomy API and SRA browser

73 (Kodama et al., 2012). Then, for each species, the pipeline retrieves a list of all

74 available RNA-seq experiments found in the SRA database and links to coding

75 sequence (CDS) files from Ensembl FTP directories (Kersey et al., 2016). The pipeline

76 then generates a tab-delimited species list table where the user can edit, add or remove

77 the links to CDS files (Table S1). Only species with CDS links specified in the table will

78 be further processed, allowing the user to define which species should be analyzed by

79 the pipeline, and also to specify alternative sources of CDS files. For each species in

80 the curated species list table, LSTrAP-Kingdom retrieves the CDS files, generates

81 kallisto index file, downloads the available fastq files (with an option for a parallel

82 download), and estimates the gene expression with kallisto (Bray et al., 2016). LSTrAP-

83 Kingdom will also download the SRA run tables for each species, containing the

84 metadata of the RNA-seq experiments used to annotate the experiments (Table S2).

In our example, we selected 116 Archaeplastida species for download. With 24

86 parallel downloads, we could download $\sim 12,000$ samples per day, when the fastq file

87 size was capped at 1GB ( 20 million reads)(Table S3). For Arabidopsis thaliana (taxid

88 3702), the mean time taken for download, gene expression estimation with kallisto, and

89 total processing is 1.41 minutes, 0.55 minutes, and 1.95 minutes, respectively (Figure

90 1B). To perform quality control of the downloaded RNA-seq, LSTrAP-Kingdom produces

91 a table describing the RNA-seq data with two parameters: $\log _{10}$-normalized number of

92 processed reads (LPR) and percentage of reads pseudo-aligned to the reference CDS

93 (PPA)(Tan et al., 2020). These parameters were used to visualize any outlier samples 
94 (Figure S1) and to recommend LPR and PPA thresholds to remove samples with a low 95 number of reads or poor mapping statistics (Table S4). Samples accepted by the user 96 (Figure S2) are used to compile a TPM matrix for each species.

\section{Automated sample annotation}

99 LSTrAP-Kingdom annotates the experiments by matching words found in the 100 experiment metadata with any organ ontology in obo format. For the Archaeplastida 101 kingdom, we used Plant Ontology's (PO) anatomical entity domain to specify plant 102 organs and tissues (Walls et al., 2019). First, for each PO term (e.g., leaves, roots, 103 seeds), the words in a PO term were stemmed (leav, root, seed), and the percentage of 104 the stemmed words of each PO found in a given RNA-seq experiment description was 105 calculated. Then, each RNA-seq sample was annotated with the PO term showing the 106 highest percentage of matched words. If multiple equal matches were found, the PO 107 term with most words was used to annotate the RNA-seq experiment.

We benchmarked the accuracy of our pipeline against manual annotation of 109 RNA-seq data from 7 species in the EVOREPRO study (Table S5, S6)(Julca et al., 110 2020). The proportion of samples that could be annotated range from $68 \%$ to $91 \%$ 111 (Figure 1C). The accuracy ranged from 46.5\% in Marchantia polymorpha to $93.3 \%$ in 112 Oryza sativa (Figure 1C). The poor accuracy in Marchantia polymorpha was due to the 113 description of the RNA-seq samples in the run table not using standard nomenclature 114 (Table S5). For example, some samples were annotated as "sporelings", a 115 developmental stage of bryophytes not represented in PO. Thus, a more 116 comprehensive PO database and a standardized sample description nomenclature will 117 improve future automated sample annotation. Thus, our automated pipeline can be 118 used to rapidly annotate thousands of RNA-seq samples (Table S7). 
121 To measure the performance of the coexpression networks generated by our pipeline,

122 we evaluated how well our networks predict genes that are components of large and 123 small ribosomal subunits (Supplementary Figure S3), as was done in a previous study

124 (Hew et al., 2020). Briefly, each ribosomal protein was functionally annotated by its 125 coexpression neighborhood functions, at a given Pearson correlation coefficient (PCC) 126 threshold and percentage of coexpression neighborhood required to annotate the 127 function. For example, a gene is annotated as a ribosomal protein if at least $50 \%$ of it's 128 coexpression neighborhood at $\mathrm{PCC}>0.7$ are ribosomal proteins. As a metric for the 129 predictive power, we used F1-score, which is a harmonic mean between the precision 130 and recall of the predictions of the coexpression network.

131 The F1-scores range from 0.8828 in Picea abies (taxid 3329) to 0.9896 in 132 Physcomitrium patens (taxid 3218) for the coexpression networks generated by 133 LSTrAP-Kingdom (Figure 1D), showing a strong predictive power of the generated 134 coexpression networks. Furthermore, the coexpression networks generated in this study 135 show slightly higher F1-scores than the networks obtained from the manually 136 constructed matrices used in the EVOREPRO study (Julca et al., 2020). This 137 demonstrates the usefulness of LSTrAP-Kingdom for kingdom-wide expression 138 analysis.

140 Funding

141 This work has been supported by the Nanyang Technological University Start-Up Grant.

142 Conflict of interest: None declared.

\section{Data Availability Statements}

145 The data underlying this article are available in the article and in its online 146 supplementary material. The RNA-sequencing data is publicly available from NCBI 147 SRA. 
Bray,N.L. et al. (2016) Near-optimal probabilistic RNA-seq quantification. Nat. Biotechnol., 34, 525-527.

Ferrari,C. et al. (2020) Expression Atlas of Selaginella moellendorffii Provides Insights into the Evolution of Vasculature, Secondary Metabolism, and Roots. Plant Cell, tpc.00780.2019.

Ferrari,C. et al. (2019) Kingdom-wide comparison reveals the evolution of diurnal gene expression in Archaeplastida. Nat. Commun., 10.

Ferrari,C. and Mutwil,M. (2019) Gene expression analysis of Cyanophora paradoxa reveals conserved abiotic stress responses between basal algae and flowering plants. New Phytol.

Hew,B. et al. (2020) LSTrAP-Crowd: prediction of novel components of bacterial ribosomes with crowd-sourced analysis of RNA sequencing data. BMC Biol., 18, 114.

Julca,I. et al. (2020) Comparative transcriptomic analysis reveals conserved transcriptional programs underpinning organogenesis and reproduction in land plants. bioRxiv, 2020.10.29.361501.

Kersey,P.J. et al. (2016) Ensembl Genomes 2016: More genomes, more complexity. Nucleic Acids Res., 44, D574-D580.

Kodama,Y. et al. (2012) The sequence read archive: explosive growth of sequencing data. Nucleic Acids Res., 40, D54-D56.

Lim,J.J.J. et al. (2020) Fungi.guru: Comparative genomic and transcriptomic resource for the fungi kingdom. Comput. Struct. Biotechnol. J., 18, 3788-3795.

Ng,J.W.X. et al. (2019) Diurnal.plant.tools: Comparative Transcriptomic and Coexpression Analyses of Diurnal Gene Expression of the Archaeplastida Kingdom. Plant Cell Physiol.

Rhee,S.Y. and Mutwil,M. (2014) Towards revealing the functions of all genes in plants. Trends Plant Sci., 19, 212-221.

Stark,R. et al. (2019) RNA sequencing: the teenage years. Nat. Rev. Genet., 20, 631656.

Tan,Q.W. et al. (2020) LSTrAP-Cloud: A User-Friendly Cloud Computing Pipeline to Infer Coexpression Networks. Genes, 11, 428.

Usadel,B. et al. (2009) Coexpression tools for plant biology: Opportunities for hypothesis generation and caveats. Plant Cell Environ., 32, 1633-1651.

Walls,R.L. et al. (2019) The Plant Ontology Facilitates Comparisons of Plant Development Stages Across Species. Front. Plant Sci., 10.

Wen Tan,Q. and Mutwil,M. (2019) Malaria.tools-comparative genomic and transcriptomic database for Plasmodium species. Nucleic Acids Res. 


\section{Supplementary materials}

Table S1. Species list for plants identified by Viridiplantae taxonomy ID (33090).

191 The table shows the taxonomic ID, species name, RNA sample count (total), RNA

sample count (Illumina), and a link to a CDS file found for each species. Links which are

193 do not belong to 'ensemblegenomes' were manually added.

Table S2. SRA runtables used for annotating the seven species.

Table S3. Logfile of download times for each species in the batch. The table shows

196 the species number, taxonomic ID, the attempt number (failed downloads are retried 197 two times), total number of downloaded fastq files, total number of failed downloads, 198 number of downloaded files in a current attempt and time taken for the current attempt.

199 Table S4. Quality control thresholds (A) recommended by LSTrAP-Kingdom and 200 (B) edited by the user. The pipeline automatically recommends cutoff thresholds for $201 \log _{10}$ processed and \% pseudoaligned and shows how many fastq files pass the given 202 threshold. The user can adjust the cutoffs to either increase or decrease the stringency.

203 Table S5. Comparison of LSTrAP-Kingdom's annotation against EVOREPRO's 204 annotation. The table shows the taxonomy ID, species name, the fastq file name (run 205 ID), EVOREPRO annotation of the sample (comprising ten categories: root, seeds, 206 stem, leaf, flower, male, female, root meristem, apical meristem, spore), PO term 207 predicted by the pipeline and outcome of the prediction (true or false). Since the 208 EVOREPRO annotation is more general (e.g., female) than the PO terms (e.g., plant 209 egg cell), we indicated where the seemingly incorrect predictions are correct in the 210 'modified annotation' column.

211 Table S6. Mapping of EVOREPRO annotation terms to the related PO terms. The 212 table used to translate the more specific PO terms to the more general EVOREPRO 213 sample annotations, as done in the 'modified annotation' column in Table S5.

214 Table S7. LSTrAP-Kingdom sample annotation for seven species. The species are 215 Marchantia polymorpha, Physcomitrium patens, Picea abies, Arabidopsis thaliana, 216 Solanum lycopersicum, Oryza sativa and Zea mays. 

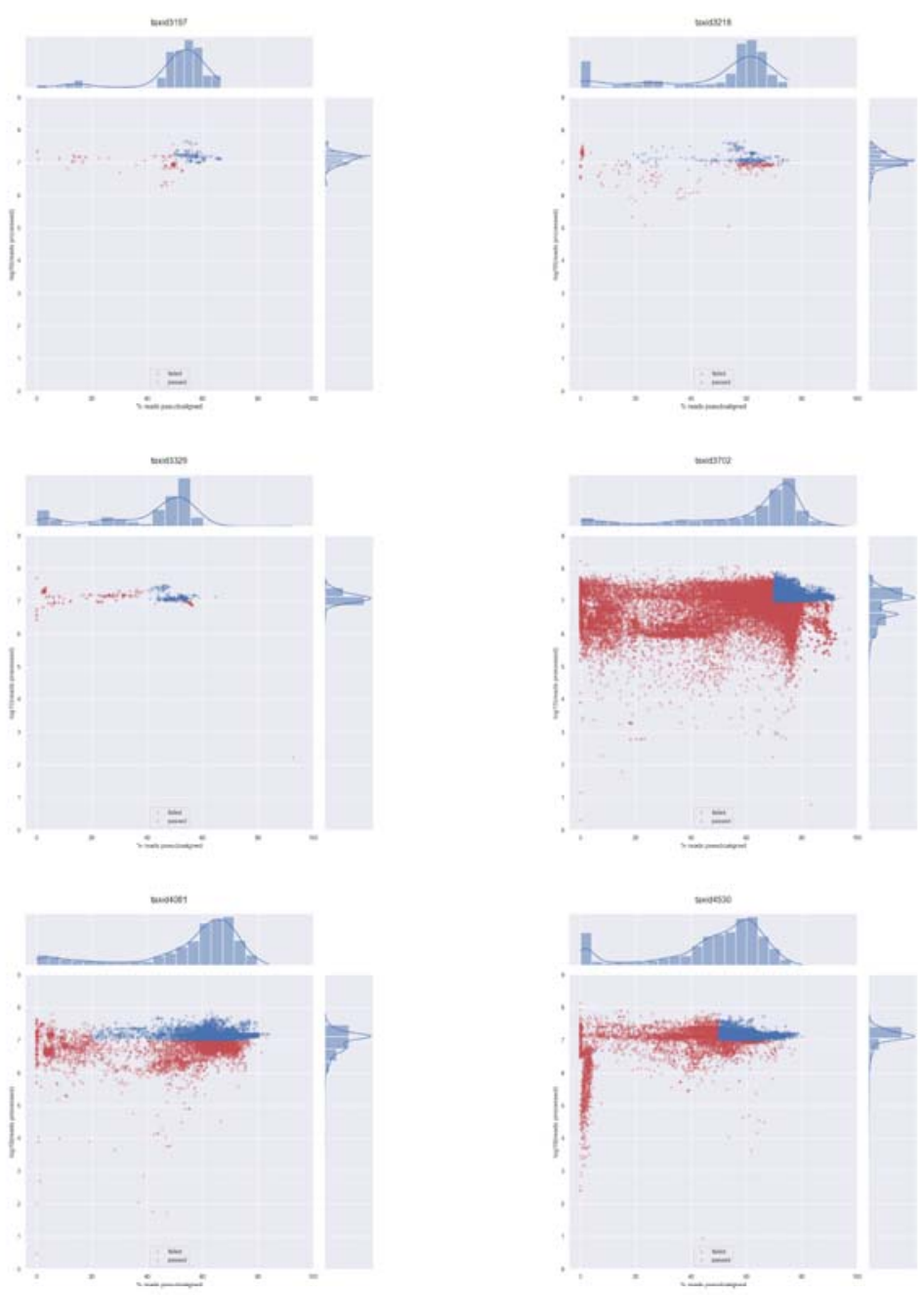

218 Figure S1. Quality control visualization with the LSTrAP-Kingdom automatically

219 suggested thresholds. Each point represents a fastq experiment, where a blue or red

220 point represents experiments that passed or failed the thresholds, respectively. The $x-$

221 and $y$-axis represent the $\%$ of pseudoaligned reads and $\log _{10}$ processed reads, 222 respectively. 
bioRxiv preprint doi: https://doi.org/10.1101/2021.01.23.427930; this version posted January 25, 2021. The copyright holder for this preprint (which was not certified by peer review) is the author/funder, who has granted bioRxiv a license to display the preprint in perpetuity. It is made available under aCC-BY-NC 4.0 International license.
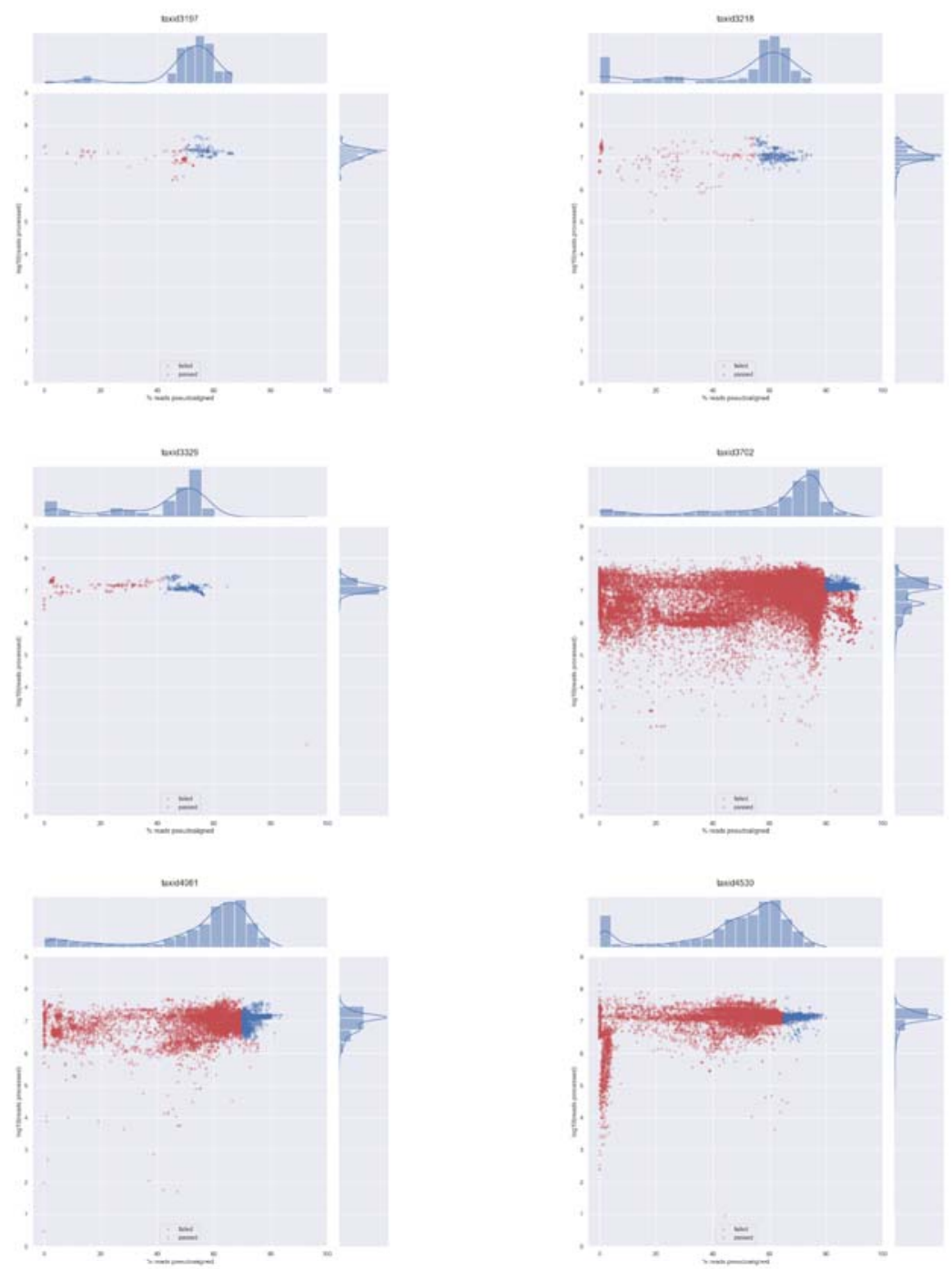

224 Figure S2. QC visualization with user-defined thresholds. Thresholds from Table 225 S4B are used in this figure. 

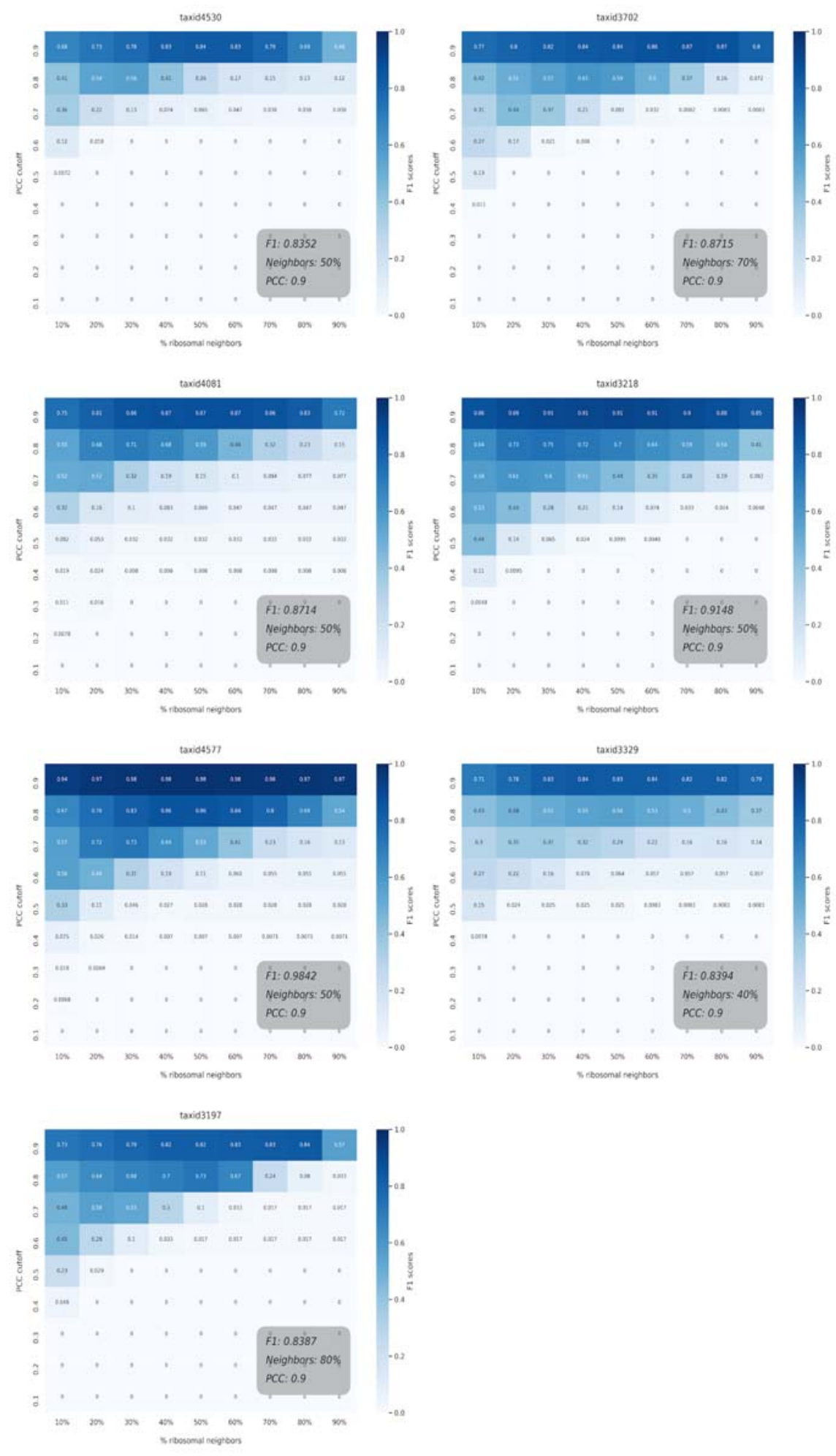

227 Figure S3: Heatmaps of F1 scores for LSTrAP-Kingdom. The heat maps indicate the 228 F1-score for each used pair of PCC and the percentage of ribosomal neighbors 229 thresholds. 